\title{
ADAPTAÇÃO TRANSCULTURAL DA ESCALA DE AVALIAÇÃO DE INCAPACIDADE EM DEMÊNCIA (DISABILITY ASSESSMENT FOR DEMENTIA - DAD)
}

\author{
Maria Tereza Carthery-Goulart ${ }^{1}$, Renata Areza-Fegyveres ${ }^{1}$, Rodrigo R. Schultz', \\ Ivan Okamoto ${ }^{2}$, Paulo Caramelli ${ }^{1}$, Paulo Henrique F. Bertolucci ${ }^{2}$, Ricardo Nitrini ${ }^{1}$
}

\begin{abstract}
RESUMO - A versão original da Escala de Avaliação de Incapacidade em Demência (Disability Assessment for Dementia, DAD) foi traduzida para a língua portuguesa e retrotraduzida para o inglês. Divergências de tradução foram identificadas e discutidas, chegando-se a uma versão que foi submetida a pré-teste para adaptação sócio-cultural. A versão final foi administrada a amostra de 29 pacientes com doença de Alzheimer provável de leve a moderada. Os coeficientes de correlação da DAD foram 0,929 e 0,932 nas avaliações inter e intra-examinadores respectivamente. Os índices de confiabilidade também foram elevados (Kappa $=0,72 ; p<0,001$ inter-examinadores e Kappa $=0,85 ; p<0,001$ intra-examinadores). A versão brasileira da escala DAD mostrou-se um instrumento de fácil aplicação e boa confiabilidade para avaliação funcional de pacientes com demência e poderá contribuir para o acompanhamento desses pacientes em nosso meio. Esta versão também poderá ser utilizada em estudos transculturais sobre habilidades funcionais de pacientes com demência.
\end{abstract}

PALAVRAS-CHAVE: demência, doença de Alzheimer, incapacidade, atividades de vida diária, diagnóstico.

\begin{abstract}
Cross-cultural adaptation of the disability assessment for dementia (DAD)
ABSTRACT - The original version of the Disability Assessment for Dementia (DAD) was translated into Portuguese and back translated to English. The divergences of translation were identified and discussed, resulting in a version that was used in a preliminary investigation for cross-cultural adaptation. The final version was administered to 29 patients with mild to moderate probable Alzheimer's disease. The correlation coefficients of DAD were 0.929 and 0.932 for the inter-examiner and test-retest evaluations respectively. The reliability indexes were also high (Kappa $0.72 p<0.001$ inter-examiners and $0.85 p<0.001$ test-retest). The Brazilian version of DAD was easy to administer and had good reliability to assess the functional status of demented patients. It will contribute to the follow-up of these patients in our population. Moreover, it can be used in transcultural studies on functional abilities in dementia.
\end{abstract}

KEY WORDS: dementia, Alzheimer's disease, disability, activities of daily living, diagnosis.

Além do comprometimento das funções cognitivas, a doença de Alzheimer (DA) também se caracteriza pelo declínio no desempenho funcional, condição necessária para o diagnóstico de acordo com os critérios do National Institute of Neurologic, Communicative Disorders and Stroke-Alzheimer's Disease and Related Disorders Association (NINCDS-ADRDA) ${ }^{1}$ e do Manual de Diagnóstico Estatístico dos Transtornos Mentais-quarta edição (DSM-IV) $)^{2}$. Muitas escalas têm sido usadas para medir o desempenho nas atividades da vida diária (AVD) na população idosa e com DA, entre elas a Avaliação de Incapacidade para Demência (DAD-Disability Assessment for Dementia), desenvolvida por Gauthier e Gélinas em $1994^{3}$. No estudo inicial, a escala demonstrou alto grau de concordância interna $(0,96)$, inter-examinadores $(0,95)$ e teste-reteste $(0,96)^{4}$.

A DAD tem como objetivos: (1) quantificar habilidades funcionais em AVD para indivíduos com déficits cognitivos, como demência; (2) qualificar as dimensões cognitivas das incapacidades nas AVD, examinando atividades básicas e instrumentais da vida diária em relação a funções executivas, o que permite a identificação de áreas problemáticas: iniciação, planejamento, organização e desempenho efetivo. A escala foi baseada no modelo de saúde proposto pela

\footnotetext{
${ }^{1}$ Grupo de Neurologia Cognitiva e do Comportamento (GNCC) da Clínica Neurológica do Hospital das Clínicas da Faculdade de Medicina da Universidade de São Paulo (HCFMUSP), São Paulo SP, Brasil; ${ }^{2}$ Setor de Neurologia do Comportamento do Departamento de Neurologia e Neurocirurgia da Escola de Paulista de Medicina, Universidade Federal de São Paulo, São Paulo SP, Brasil (EPM-UNIFESP).
}

Recebido 22 Setembro 2007, recebido na forma final 11 Maio 2007. Aceito 19 Junho 2007. 
Organização Mundial de Saúde, que define incapacidade funcional como qualquer restrição na habilidade de executar uma atividade, tarefa ou comportamento da vida diária. A DAD inclui a avaliação de atividades básicas, instrumentais e de lazer. As atividades básicas da vida diária (importantes para o autocuidado) incluem a capacidade de se vestir, realizar higiene pessoal, controle esfincteriano e alimentação. As atividades instrumentais (importantes para manutenção em um ambiente específico) incluem a capacidade de preparar pequenas refeições, realizar trabalhos domésticos, cuidados com finanças e correspondências, sair, tomar remédios e ficar em casa de forma segura, itens que podem ser cruciais para o diagnóstico de um estágio precoce da $\mathrm{DA}^{5,6}$. Além de avaliar a realização efetiva de atividades de lazer, a escala inclui questões sobre o interesse mostrado por elas. A DAD tem sido utilizada em ensaios clínicos, tendo sido capaz de evidenciar o efeito positivo de drogas sobre o desempenho funcional dos pacientes (manutenção ou melhora nas AVD após tratamento) $)^{7-17}$. Considerando esses aspectos, realizamos a tradução e adaptação deste instrumento para a língua portuguesa, verificando a confiabilidade interexaminadores e teste-reteste da versão traduzida e adaptada.

\section{MÉTODO}

Utilizamos as normas propostas por Guillemin et al. ${ }^{18}$ para adaptação transcultural da escala. A versão original da DAD foi traduzida para o português por uma empresa especializada em tradução de textos médicos. Esta versão foi em seguida traduzida de volta para o inglês, de modo independente, por dois neurologistas experientes em escalas para demência e fluentes em inglês. As diferenças de tradução foram identificadas e discutidas em painel, considerando as razões para as diferenças e comparando-as com a forma original em Inglês, chegando-se a uma versão que foi submetida a um pré-teste para adaptação sócio-cultural. Nesta fase, oito indivíduos atendidos pelo Grupo de Neurologia Cognitiva e do Comportamento da Clínica Neurológica do Hospital das Clínicas da Faculdade de Medicina da Universidade de São Paulo (GNCC-HCFMUSP), que preencheram os critérios diagnósticos do NINCDS-ADRDA ${ }^{1}$ para DA provável, foram avaliados e seus cuidadores entrevistados. Durante a aplicação dos questionários, solicitou-se que cada cuidador explicasse o que tinha entendido de cada pergunta. A partir deste levantamento, foi elaborado um texto introdutório explicando as características das questões da escala, foram feitas pequenas adaptações de linguagem e propostos exemplos padronizados para as questões que geraram dúvidas, chegando-se à versão final que foi administrada a uma amostra de 29 cuidadores de pacientes com diagnóstico de DA provável de leve a moderada, de acordo com os critérios diagnósticos do NINCDSADRDA $^{1}$ e do DSM III-R ${ }^{19}$ respectivamente, atendidos nos ambulatórios do GNCC-HCFMUSP e do Setor de Neurologia do Comportamento da Universidade Federal de São Paulo. As entrevistas foram realizadas com os cuidadores nos ambulatórios recém mencionados, nos dias de consulta de acompanhamento de tais pacientes. A Tabela apresenta as características demográficas dos pacientes avaliados. Vinte e quatro pacientes fizeram parte da amostra para checar a confiabilidade inter-examinadores e 22 pacientes para checar a confiabilidade teste-reteste.

A versão final da escala, incluindo as normas de aplicação está disponível em www.abneuro.org/departamento cientifico/Neurologia Cognitiva e do Envelhecimento.

Análise estatística - Para determinar os coeficientes de correlação nas avaliações inter e intra-examinadores utilizamos o teste não-paramétrico de Spearman. Além disso, a confiabilidade foi avaliada calculando-se o valor de $K a$ ppa, que indica o grau de concordância do resultado de um teste quando realizados por dois examinadores (interexaminadores) e pelo mesmo examinador em dois momentos (teste-reteste).

O estudo foi aprovado pela Comissão de Ética para Análise de Projetos de Pesquisa da Diretoria Clínica do Hospital das Clínicas e da Faculdade de Medicina da Universidade de São Paulo e pelo Comitê de Ética em Pesquisa da Universidade Federal de São Paulo. Todos os participantes assinaram o Termo de Consentimento Informado.

\section{RESULTADOS}

A versão final da escala foi de fácil aplicação e entendimento, levando aproximadamente 20 minutos para ser administrada.

Os coeficientes de correlação da DAD foram 0,929 e 0,932 nas avaliações inter e intra-examinadores respectivamente. Os índices de confiabilidade também foram elevados (Kappa $=0,72 ; p<0,001$ inter-examinadores e Kappa $=0,85 ; p<0,001$ intra-examinadores). A pontuação média obtida na primeira avaliação foi 70,5 e desvio-padrão 14,72 .

\section{DISCUSSÃO}

A DAD mostrou-se um instrumento útil e de fácil administração que poderá ser utilizado para monitorar a progressão de quadros demenciais tanto na atividade clínica como em pesquisa. Além disso, por ser uma escala utilizada em vários países, a versão brasi-

Tabela. Características demográficas dos pacientes avaliados.

\begin{tabular}{lc}
\hline Características & Pacientes (29) \\
\hline Sexo & $14 \mathrm{M} 15 \mathrm{~F}$ \\
Idade - média (DP) & $74,1(8,7)$ \\
Escolaridade - média (DP) & $6,8(4,8)$ \\
MEEM - média (DP) & $19,9(3,8)$ \\
\hline
\end{tabular}

$M$, masculino; $F$, feminino; DP, desvio-padrão; MEEM, Mini-Exame do Estado Mental. 
leira possibilitará estudos transculturais sobre estes aspectos. A versão traduzida e adaptada, mostrou-se uma escala confiável, em relação à reprodutibilidade tanto pelo mesmo examinador em dois momentos distintos, quanto entre diferentes examinadores. A validação realizada na China teve também índices de correlação entre-examinadores e teste-reteste bastante elevados. No entanto, neste trabalho houve necessidade de se aumentar o número de questões na adaptação do instrumento ${ }^{20}$.

Entretanto, é importante comentar que a escala avalia efetivamente o que o paciente é capaz de fazer, não quantificando ou caracterizando áreas em que ele começa a ter dificuldades. Por exemplo, se o paciente conseguiu realizar determinada atividade uma única vez nas últimas duas semanas sem ajuda e sem ser lembrado, ele obtém pontos nessa tarefa, não importando se em dez tentativas apenas uma foi efetiva. Por isso, é interessante fazer anotações quando o paciente começa a ter dificuldades para um monitoramento mais preciso.

A avaliação das AVD em pacientes com demência oferece vantagens como: melhorar a acurácia diagnóstica, avaliar o efeito de drogas em ensaios clínicos, obter informações sobre condições, habilidades e limitações que não são regularmente avaliadas na prática clínica, auxiliar a determinar o prognóstico e avaliar e planejar a necessidade de cuidados ${ }^{21}$. A avaliação da incapacidade é necessária para estabelecer a gravidade da doença, pois os critérios diagnósticos, como os do DSM III- $\mathrm{R}^{19}$, requerem a caracterização de habilidades perdidas e mantidas ${ }^{22}$. $O$ estabelecimento de estágios pode ajudar a estimar a necessidade de suporte das famílias e em nível populacional, estimar cuidados institucionais, políticas sociais e de serviços ${ }^{23}$.

Um dos problemas das avaliações baseadas nos informantes é que os mesmos tendem a superestimar a incapacidade funcional e alguns deles podem não estar suficientemente informados para fazer a avaliação ${ }^{21,24}$. Além disso, seu estado psicológico interfere nas respostas ${ }^{23}$. No entanto, estes instrumentos possibilitam obter informações sobre pacientes resistentes à avaliação, ou que não podem mais ser testados. Adicionalmente, podem ser administrados via correspondência ou telefone, apresentam maior facilidade de adaptação transcultural e são menos influenciados pela escolaridade que testes neuropsicológicos $^{24}$. As desvantagens podem ser contornadas, verificando-se quantas horas o informante passa com o paciente e o tipo de cuidado que é administrado.
A incapacidade nas AVD tem um grande impacto sobre a qualidade de vida dos pacientes e de seus cuidadores ${ }^{7}$, sendo um aspecto que prediz mais fortemente o uso de serviços de saúde e a institucionalização, mais do que outras medidas de gravidade ${ }^{25}$. Na amostra estudada, o questionário sensibilizou alguns cuidadores quanto a questões preservadas, permitindo orientações para o aumento da autonomia e qualidade de vida do paciente. Em outros casos, também apontou aspectos negligenciados nos cuidados, sobretudo no que se refere à higiene.

Em conclusão, a versão brasileira da escala DAD mostrou-se um instrumento de fácil aplicação na amostra avaliada, com boa confiabilidade tanto inter quanto intra-examinadores. Além disso, pode contribuir para a avaliação funcional, o acompanhamento de pacientes com demência no Brasil, e para a inclusão de nosso país em projetos de cooperação internacional que utilizem esta escala, assim como em estudos transculturais.

\section{REFERÊNCIAS}

1. McKhann G, Drachman D, Folstein M, Katzman R, Price D, Stadlan EM. Clinical diagnosis of Alzheimer's disease: report of the NINCDSADRDA work group. Neurology 1984;34:939-944.

2. American Psychiatric Association. Diagnostic and statistical manual of mental disorders. 4.Ed. Washington (DC): APA, 1994

3. Gauthier L, Gélinas I, McIntyre M, Gauthier S, Laberge H, Dauphinee SW. Disability Assessment for Dementia (DAD) user's guide, 1994.

4. Gélinas I, Gauthier L, McIntyre M, Gauthier S. Development of a functional measure for persons with Alzheimer's disease: the disability assessment for dementia. Am J Occup Ther 1999;53:471-481.

5. Gauthier L, Gauthier S. Assessment of functional changes in Alzheimer's disease. Neuroepidemiology 1990;9:183-188.

6. Gauthier S, Gélinas I, Gauthier L. Functional disability in Alzheimer's disease. Int Psychogeriatr 1997;9:163-165.

7. Feldman H, Sauter A, Donald A, et al. The disability assessment for dementia scale: a 12-month study of functional ability in mild to moderate severity Alzheimer disease. Alzheimer Dis Assoc Disord 2001;15:89-95.

8. Feldman HH, Van Baelen B, Kavanagh SM, Torfs KE. Cognition, function, and caregiving time patterns in patients with mild-to-moderate Alzheimer disease: a 12 month analysis. Alzheimer Dis Assoc Disord 2005;19:29-36.

9. Suh GH, Ju YS, Yeon BK, Ahah A. A longitudinal study of Alzheimer's disease: rates of cognitive and functional decline. Int J Geriatric Psychiatry 2004;19:817-824.

10. Feldman H, Gauthier S, Hecker J, Vellas B, Subbiah P, Whalen E. A 24week, randomized, double-blind study of donepezil in moderate to severe Alzheimer's disease. Neurology 2001;57:613-620.

11. Feldman H, Gauthier S, Hecker J, et al. Donepezil MSAD Study Investigators Group. Efficacy of donepezil on maintenance of activities of daily living in patients with moderate to severe Alzheimer's disease and the effect on caregiver burden. J Am Geriatr Soc 2003;51:737-744.

12. Wilcock GK, Lilienfeld S, Gaens E. Efficacy and safety of galantamine in patients with mild to moderate Alzheimer's disease: multicentre randomised controlled trial. Galantamine International-1 Study Group. BMJ 2000;321:1445-1449.

13. Orgogozo JM, Small GW, Hammond G, Van Baelen B, Schwalen S. Effects of galantamine in patients with mild Alzheimer's disease. Curr Med Res Opinion 2004;20:1815-1820.

14. Farlow MR, Cyrus PA. Metrifonate therapy in Alzheimer's disease: a pooled analysis of four randomized, double-blind, placebo-controlled trials. Dement Geriatr Cogn Dis 2000;11:202-211.

15. Karaman Y, Erdogan F, Köseoglu, Turan T, Ersoy AO. A 12-month study of the efficacy of rivastigmine in patients with advanced moderate Alzheimer's disease. Dem Geriatric Cogn Dis 2005;19:51-56. 
16. Reisberg B, Doody R, Stoffler A, Schmitt F, Ferris S, Mobius HJ. Memantine study group. Memantine in moderate-to-severe Alzheimer's disease, N. Eng. J. Med 2003;348:1333-1341.

17. Tariot PN, Farlow MR, Grossberg GT, Graham SM, McDonald S, Gergel I. Memantine Study Group. Memantine treatment in patients with moderate to severe Alzheimer disease already receiving donepezil: a randomized controlled trial. JAMA 2004; 291:317-324.

18. Guillemin F, Bombardier C, Beaton D. Cross-cultural adaptation of health-related quality of life measures: literature review and proposed guidelines. J Clin Epidemiol 1993;46:1417-1432.

19. American Psychiatric Association. Diagnostic and statistical manual of mental disorders. 3.Ed. rev. Washington (DC): APA, 1987.

20. Mok CC, Siu AM, Chan WC, Yeung KM, Pan PC, Li SW. Functional disabilities profile of chinese elderly people with Alzheimer's disease: a validation study on the chinese version of the disability assessment for dementia. Dement Geriatr Cogn Disord 2005;20:112-119.

21. Desai AK, Grossberg GT, Dharmesh NS. Activities of daily living in patients with dementia: clinical relevance, methods of assessment and effects of treatment. CNS Drugs 2004;18:853-875.

22. Jorm AF. Disability in dementia: assessment, prevention, and rehabilitation. Disabil Rehab 1994;16:98-109.

23. Zanetti O, Frisoni GB, Rozzini L, Bianchetti A, Trabucchi M. Validity of direct assessment of functional status as a tool for measuring Alzheimer's disease severity. Age and Ageing 1998;27:615-622.

24. Jorm AF. Assessment of cognitive impairment and dementia using informant reports. Clin Psychol Rev 1996;16:51-73.

25. Canadian Study of Health and Aging Working Group. Patterns of caring for people with dementia in Canada. Can J Aging 1994;13:470-487. 